\title{
Gliosarcomas: Analysis of Braga Hospital Cases and Review of the Literature
}

\author{
Rui Manuel Almeida Ramos ${ }^{1 *}$, Maria João Machado1, Hugo Pereira ${ }^{1}$, Renata Marques ${ }^{1}$, Cristiano \\ Antunes', Ricardo Moreira', Afonso Almeida Pinto', Marta Almeida', Júlia Amorim ${ }^{1}$, Carlos \\ Alegria ${ }^{1}$, Rui Almeidla ${ }^{1}$ and Vânia Magalhães ${ }^{2}$ \\ ${ }^{1}$ Department of Neurosurgery, Braga Hospital, Portugal \\ ${ }^{2}$ EPI Unit, Institute of Public Health, University of Porto, Portugal
}

${ }^{*}$ Corresponding author: Rui Manuel Almeida Ramos, Department of Neurosurgery, Braga Hospital, Sete Fontes, São Vitor, Braga, Portugal, Tel: 351253027000; E-mail: ruimramos@hotmail.com

Rec date: December 14, 2018; Acc date: January 25, 2019; Pub date: February 06, 2019

Copyright: (c) 2019 Ramos RMA, et al. This is an open-access article distributed under the terms of the Creative Commons Attribution License, which permits unrestricted use, distribution, and reproduction in any medium, provided the original author and source are credited.

Citation: Ramos RMA, Machado MJ, Pereira H, Marques R, Antunes C, et al. (2019) Gliosarcomas: Analysis of Braga Hospital Cases and Review of the Literature. Neurooncol Open Access Vol.4 No.1:1.

\section{Abstract}

Background: Gliosarcomas are very rare tumors of central nervous system, as such, the data of the literature are scarce, which makes its characterization and therapeutic approach more difficult.

Methods: A survey about gliosarcomas in Hospital de Braga was carried out between January 1995 and October 2013. A bibliographic review was also carried out.

Results: We get a total of 18 cases and the main aim of this study was to evaluate survival according to different variables (age, gender, time symptoms to diagnose, karnofsky, MRI necrosis, MRI mass effect, MRI edema, proximity to the dura-mater, lobar location, hemispheric location, tumor size, gliosarcoma type, macroscopy, type of resection, adjuvant treatment and metastization). The factors associated with a better survival were: age inferior to 50 years old, female gender, karnofsky $>80$, time symptoms to diagnose $<1$ month, necrosis $<50 \%$ tumor volume, midline shift $<0.5 \mathrm{~cm}$, edema $<$ tumor volume, tumor not adjacent to the dura-mater, size $<5 \mathrm{~cm}$, occipital lobe, left hemisphere, primary type, macroscopy similar to glioblastoma, radical resection, adjuvant RT, non metastization. There were two factors with influence on survival and statistically significant $p$ value: midline shift less than $0.5 \mathrm{~cm}$ and location in the left hemisphere.

Conclusion: This sample presents some factors with effect on the survival and that can be taken into account in the approach of these tumors.

Keywords: Brain tumor; Gliosarcoma; Radiotherapy; Chemotherapy; Survival

\section{Introduction}

Gliosarcomas are rare neoplasms of the central nervous system, constituting $0.48 \%$ of intracranial tumors and $1.8-10 \%$ of glioblastomas [1-9]. They were first described in 1895 as brain neoplasms formed by glial and mesenchymal components and in 2000 the World Health Organization (WHO) classified them as a variant of glioblastomas. The current definition is based on the WHO classification of 2016, considering them as part of the IDH-wildtype glioblastomas spectrum.

As they are rare tumors, the data of the literature are scarce, which makes its characterization and therapeutic approach more difficult. This study aims to present the case series of gliosarcomas at the Hospital de Braga between January 1995 and October 2013 and to perform a statistical analysis of the different variables. A bibliographic review was also carried out.

\section{Literature Review}

It was carried out a survey of the clinical processes with histological diagnosis of gliosarcoma in the database of the Anatomic Pathology Service of Braga Hospital between January 1995 and October 2013 in the adult population. By that time, routine genetic study was not performed at our hospital.

Later, an analysis of these processes was performed, which evaluated the following parameters: age, sex, race, comorbidities, clinic that motivated investigation, time elapsed between the beginning of the clinic and the imaging diagnosis, preoperative karnofsky scale, imaging on computed tomography (CT) and magnetic resonance imaging (MRI), location, type (primary, secondary or after intracranial radiotherapy), macroscopic appearance observed during surgery, type of resection, adjuvant treatment, response to treatment, time to recurrence, survival, occurrence of metastization and in the secondary the time between 
diagnosis of glioblastoma and gliosarcoma. Survival was then evaluated according to the different variables.

Primary gliosarcomas were considered as those that appeared without previous diagnosis of intracranial neoplasia and with an initial histological result compatible with gliosarcoma. Secondary gliosarcomas were considered to meet the following criteria: Patients with prior diagnosis of intracranial malignant glioma without gliosarcoma components and subsequent lesion recurrence with histological diagnosis of gliosarcoma. Gliosarcomas were also considered after intracranial radiotherapy. The latter occur in patients submitted to intracranial radiotherapy for other brain neoplasms, but without previous diagnosis of glioblastoma.

The imaging characteristics were analyzed preferably in the cerebral MRI, or when it was not available according to the CT. The size of the lesion was characterized according to the largest diameter in the axial sections. The remaining characteristics were evaluated in the brain MRI according to the Hammoud scale [10], evaluating necrosis, mass effect, contrast uptake and surrounding edema.

Macroscopy refers to the characteristics of the lesion observed by the Neurosurgeon during the surgical intervention. The meningioma-like tumors were adhered to the dura-mater, with hard consistency and a cleavage plane. Those similar to glioblastoma were infiltrative tumors, with necrotic areas and poorly defined limits. The resection was classified as radical or partial according to the absence or presence of residual tumor in the postoperative control imaging examination.

The histological diagnosis was based on WHO 2007 criteria, considering the existence of two sets of morphologically distinct cells in Hematoxylin Eosin. One with glial morphology of the gliomatous component, the other with fusiform cells corresponding to the sarcomatous region.

The primary objective of the statistical analysis was to evaluate survival according to different variables. In cases where it was not possible to know the exact date of death or in the case of patients still alive, the survival was underestimated by the last hospital record. For the statistical analysis of the data, the Social Package for Social Sciences (SPSS) version 24 was used. The test used for the survival analysis according to the different variables was the test for independent samples (in the presence of normal distribution), and the Chi-square test (in the absence of a normal distribution). A statistically significant difference was considered for values of $p<0.05$.

The literature review was performed in the PubMed database with the terms "gliosarcoma", "gliosarcoma", "gliosarcomas" and "gliosarcomas". The most relevant results were then compared with our series.

\section{Results}

The sample included 18 cases of gliosarcomas, which corresponded to $5 \%$ of all glioblastomas during the same period of time. An increase in the incidence of gliosarcomas has been observed in the last years of the study (Graph 1).

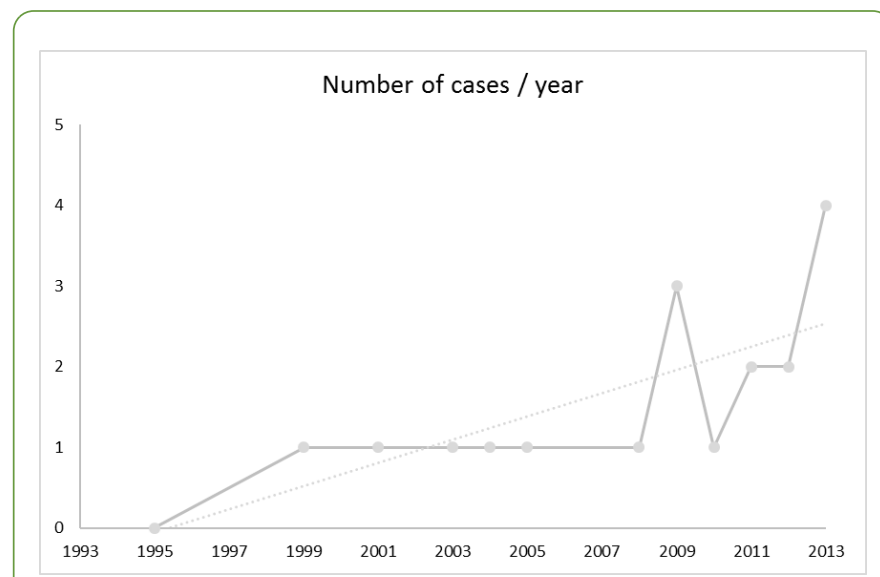

Graph 1 Number of gliosarcomas per year in Braga Hospital.

The clinical characteristics are summarized in Table 1. All patients were Caucasian, $61 \%$ male and $39 \%$ female, aged 24-80 years (mean age of 56 years). The female: male ratio was 1.56:1.

Table 1 Characteristics of Gliosarcoma at Braga Hospital.

\begin{tabular}{|c|c|c|c|c|c|c|c|c|c|c|c|}
\hline $\begin{array}{l}\text { Cas } \\
\text { e }\end{array}$ & $\begin{array}{l}\text { Se } \\
x\end{array}$ & $\begin{array}{l}\text { Age } \\
\text { (Years) }\end{array}$ & Location & $\begin{array}{l}\text { Typ } \\
\text { e }\end{array}$ & $\begin{array}{l}\text { Tyme GB } \\
\text { to GS } \\
\text { (Months) }\end{array}$ & Macroscopy & Resection & $\begin{array}{l}\text { Adjuvant } \\
\text { treatment }\end{array}$ & $\begin{array}{l}\text { Relapse } \\
\text { (Months) }\end{array}$ & $\begin{array}{l}\text { Survival } \\
\text { (Months) }\end{array}$ & Metastization \\
\hline 1 & $M$ & 60 & F left & $1^{\circ}$ & - & Glioblastoma & $\mathrm{P}$ & $\mathrm{RT}+\mathrm{CT}$ & 21 & 25 & No \\
\hline 2 & $\mathrm{~F}$ & 56 & T left & $2^{\circ}$ & 11 & NA & $\mathrm{P}$ & CT & 3 & 13 & No \\
\hline 3 & $\mathrm{~F}$ & 63 & F left & $1^{\circ}$ & - & Meningioma & $\mathrm{R}$ & CT & 4 & 13 & No \\
\hline 4 & $\mathrm{~F}$ & 69 & T left & $2^{\circ}$ & 24 & Glioblastoma & $\mathrm{R}$ & $\mathrm{N}$ & 3 & 10 & No \\
\hline 5 & $M$ & 72 & T left & $2^{\circ}$ & 9 & NA & $\mathrm{R}$ & $\mathrm{N}$ & 6 & 11 & No \\
\hline 6 & $\mathrm{~F}$ & 56 & T right & $1^{\circ}$ & - & Meningioma & $\mathrm{R}$ & $\mathrm{RT}+\mathrm{CT}$ & 8 & 9 & No \\
\hline 7 & $\mathrm{~F}$ & 71 & Tright & $1^{\circ}$ & - & NA & $\mathrm{P}$ & $\mathrm{RT}$ & 2 & 7 & No \\
\hline 8 & $M$ & 42 & T right & $1^{\circ}$ & - & NA & $\mathrm{P}$ & $\mathrm{RT}+\mathrm{CT}$ & NA & 2 & No \\
\hline
\end{tabular}




\begin{tabular}{|c|c|c|c|c|c|c|c|c|c|c|c|}
\hline 9 & M & 56 & Tright & $1^{\circ}$ & - & NA & $\mathrm{P}$ & $\mathrm{N}$ & 2 & 2 & No \\
\hline 10 & $M$ & 58 & T left & $1^{\circ}$ & - & NA & $\mathrm{R}$ & $\mathrm{RT}+\mathrm{CT}$ & 5 & 9 & Yes \\
\hline 11 & $\mathrm{~F}$ & 60 & $P$ left & $1^{\circ}$ & - & NA & $\mathrm{P}$ & $\mathrm{RT}+\mathrm{CT}$ & 5 & 17 & No \\
\hline 12 & $M$ & 24 & T left & $2^{\circ}$ & 16 & NA & $\mathrm{R}$ & CT & 5 & 9 & No \\
\hline 13 & $M$ & 48 & T left & $1^{\circ}$ & - & Glioblastoma & $\mathrm{R}$ & $\mathrm{RT}+\mathrm{CT}$ & 6 & 14 & No \\
\hline 14 & $M$ & 44 & F left & $1^{\circ}$ & - & NA & $\mathrm{R}$ & $\mathrm{N}$ & $\begin{array}{l}\text { Post-op } \\
\text { death }\end{array}$ & $\begin{array}{l}\text { Post-op } \\
\text { death }\end{array}$ & No \\
\hline 15 & $M$ & 55 & F right & $2^{\circ}$ & 8 & NA & $\mathrm{R}$ & $\mathrm{N}$ & 2 & 2 & No \\
\hline 16 & $\mathrm{~F}$ & 46 & O left & $1^{\circ}$ & & NA & $\mathrm{R}$ & RT & 24 & 31 & No \\
\hline 17 & $M$ & 80 & T left & $1^{\circ}$ & - & NA & ND & $\mathrm{N}$ & 5 & 11 & No \\
\hline 18 & $M$ & 51 & T right & $1^{\circ}$ & - & NA & $\mathrm{P}$ & $\mathrm{N}$ & 3 & 4 & No \\
\hline \multicolumn{12}{|c|}{ Abbreviations: GB: Glioblastoma; GS: Gliosarcoma; NA: Not Available; F: Front; T: Temporal; O: Occipital; P: Parietal; R: Radical; P: Partial; RT: Radiotherapy; } \\
\hline
\end{tabular}

The most frequent comorbidities were cardiovascular risk factors in 11 patients, and 7 patients had no comorbidities. In addition to the cardiovascular risk factors, 2 patients had a history of depression concomitantly. The most frequent clinical presentations were headache, dysphasia and behavioral changes. In relation to the time elapsed between the beginning of the clinic and the imaging diagnosis the average was 17 days. The preoperative karnofsky scale was divided equally by 90,80 and 70 points (33.3\%).

In 9 of the 18 patients, preoperative evaluation with brain CT was not possible. In the others, the main characteristic was a heterogeneous lesion with linear contrast uptake at the periphery. In 2 of the 18 patients it was not possible to access the preoperative cerebral MRI. In the others, the most frequent findings were necrosis greater than $50 \%$ of the tumor volume, midline shift greater than $0.5 \mathrm{~cm}$, marked increase of the signal with contrast and edema equal to tumor volume. In $76.9 \%$ of cases the tumor was adjacent to the dura mater. The size of the tumor lesion varied between 3 and $9 \mathrm{~cm}$ with an average of $5.7 \mathrm{~cm}$.

The most frequently brain lobe involved was temporal $(66.7 \%)$, followed by the frontal lobe $(16.7 \%)$. The most frequent hemispheric location was left (66.7\%). Primary gliosarcomas corresponded to $72.2 \%$ of the gliosarcomas in this series, being the remaining the secondary type. There were no cases of gliosarcoma after intracranial radiation therapy.

In secondary gliosarcomas, the mean time elapsed between surgery to glioblastoma and surgery to gliosarcoma was 13.6 months with a minimum of 8 and a maximum of 24 months. The macroscopic aspect of the gliossarcomas observed during the surgery were not described in $72.2 \%$. Macroscopy similar to glioblastoma were reported in $16.7 \%$ and similar to meningioma in $11.1 \%$. The type of resection in the first surgery of the gliosarcoma was radical in $55.6 \%$, partial in $38.9 \%$ and unknown in $5.6 \%$.
Regarding the adjuvant treatment, $38.9 \%$ of the gliosarcomas were not submitted to any adjuvant treatment, $33.3 \%$ were submitted to radiotherapy and chemotherapy, $16.7 \%$ to isolated chemotherapy and $11.1 \%$ to isolated radiotherapy. Response to treatment was unknown in $5.9 \%$. In the remaining patients, there was tumor recurrence and 3 patients underwent a new surgery. The mean time to relapse was 6.5 months, with a minimum of 2 and a maximum of 24 months. The median survival after the histological diagnosis of gliosarcoma was 11.1 months, with a minimum of 2 and a maximum of 31 months. Only 1 of the 18 cases metastasized (5.6\%) and 1 of the patients died in the immediate postoperative period due to hemorrhage of the surgical site (5.6\%). The most frequent characteristics of the gliosarcomas of Hospital de Braga are summarized in Table 2 (Figure 1).

Table 2 Most frequent characteristics of Braga Hospital gliosarcomas.

\begin{tabular}{|l|}
\hline Patient characteristics \\
\hline 56 years old \\
\hline Male gender \\
\hline Cardiovascular risk factors \\
\hline Clinical presentation with headaches, behavior changes and dysphasia \\
\hline Time symptoms to diagnosis (weeks) $=3$ \\
\hline Imaging features \\
\hline CT: Heterogeneous lesion with linear periphery contrast enhancement \\
\hline $\begin{array}{l}\text { MRI: Necrosis }>50 \% \text { of tumor volume, midline shift }>0.5 \mathrm{~cm}, \text { marked increase } \\
\text { of signal with contrast and edema equal to tumor volume }\end{array}$ \\
\hline Tumor adjacent to the dura mater \\
\hline Size (cm) $=5.7$ \\
\hline Temporal lobe \\
\hline \begin{tabular}{l} 
Left hemisphere \\
\hline
\end{tabular} \\
\hline
\end{tabular}


Other characteristics

Primary type macroscopy similar to glioblastoma

Radical resection

Relapse $($ months $)=6.5$

Medium survival $($ months $)=11.1$

Metastization $=5.6 \%$

Time between glioblastoma and gliosarcoma (months)=13.6
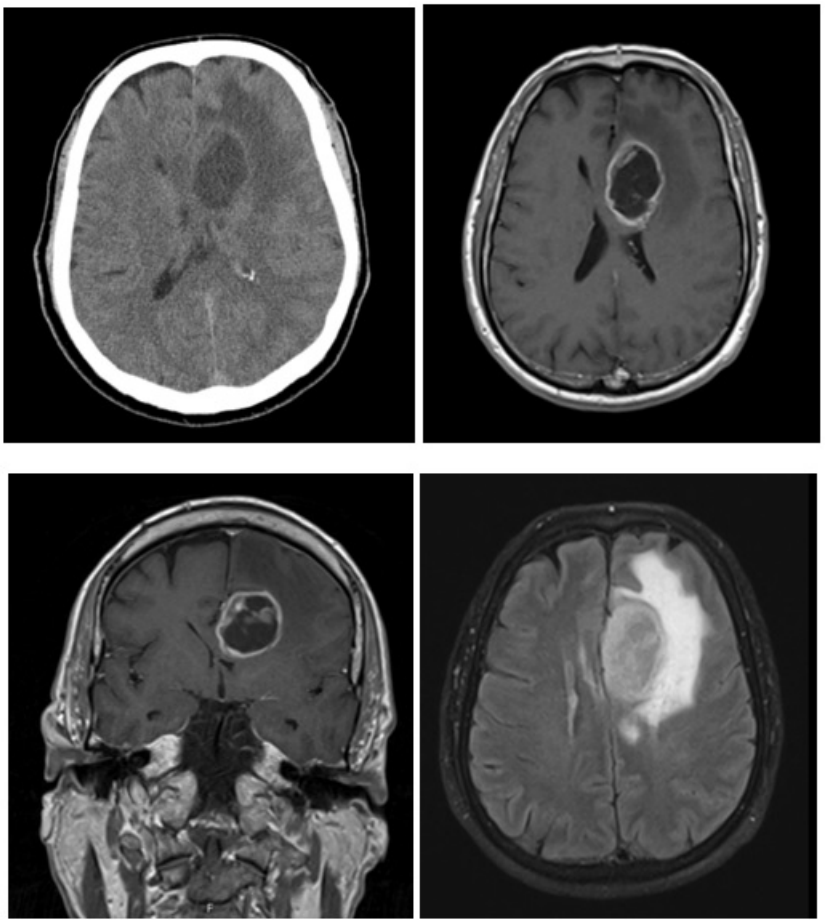

Figure 1 Images of Case 1 of Table 2. Upper left image corresponds to $\mathrm{CT}$ without contrast; right upper image to axial T1 MRI with contrast; left inferior image to coronal T1 MRI with contrast; right inferior image to MR in FLAIR. It is visible a massive intra-parenquimatous left frontal lesion with cingulate and corpus callosum involvement, heterogenous contrast uptake and marked vasogenic edema.
A survival analysis was summarized in Table 3 . It was verified that individuals aged 50 years or older obtained an average survival of 10.2 months compared to 11.2 months for age less than 50 years. Males were associated with worse survival, 8.1 months, compared to 14.3 months for females.

The time between symptoms and diagnosis, equal to or greater than one month was associated with lower survival (8.8 months) compared to time between clinical and diagnosis less than one month (11.4 months). Patients with preoperative karnofsky less than 80 were associated with a shorter survival, 7.4 months compared with 13.5 months for karnofsky equal to or greater than 80 .

Analyzing the survival according to the necrosis in MRI, patients with necrosis equal to or greater than $50 \%$ of the tumor volume obtained lower survival than those with necrosis of less than $50 \%$ of the tumor volume (9.8 and 12.1 months respectively). Patients with mean midline shift equal to or greater than $0.5 \mathrm{~cm}$ survived less than those with shift less than $0.5 \mathrm{~cm}$ ( 7.6 months and 16.3 respectively). Patients with MRI edema equal to or greater than tumor volume had a much lower survival rate than those with edema less than tumor volume (5.0 and 12.3 months respectively). Tumors adjacent to the dura were associated with lower survival rates (9.3 months versus 14.8 months for non-adherents). A parietal lobe localization acquired the best survival (17.0 months) and a shorter one for temporal location (8.4 months), as well as left hemisphere lesions obtained better survival (13.6 months) than lesions in the right hemisphere ( 4.3 months). Lesions with a size equal to or greater than $5 \mathrm{~cm}$ had lower survival rates compared to those of less than $5 \mathrm{~cm}$ (7.7 and 13.3 months respectively).

The time between symptoms and diagnosis, equal to or greater than one month was associated with lower survival (8.8 months) compared to time between clinical and diagnosis less than one month (11.4 months). Patients with preoperative karnofsky less than 80 were associated with a shorter survival, 7.4 months compared with 13.5 months for karnofsky equal to or greater than 80 .

Table 3 Survival analysis.

\begin{tabular}{|l|l|l|}
\hline Variables & Groups & Survival (months) \\
\hline Age (years) & $>=50$ & 10.2 \\
\hline & 11.2 \\
\hline Gender & Male & 8.1 \\
\hline & Female & 14.3 \\
\hline Time symptoms to diagnose (months) & $>=1$ & 8.8 \\
\hline & $<1$ & $<1.4$ \\
\hline
\end{tabular}




\begin{tabular}{|c|c|c|}
\hline Karnofsky & $>=80$ & 13.5 \\
\hline & $<80$ & 7.4 \\
\hline \multirow[t]{2}{*}{ MRI necrosis ( $\%$ of tumor volume) } & $>=50$ & 9.8 \\
\hline & $<50$ & 12.1 \\
\hline \multirow[t]{2}{*}{ MRI mass effect (cm of midline shift) } & $>=0.5$ & 7.6 \\
\hline & $<0.5$ & 16.3 \\
\hline \multirow[t]{2}{*}{ MRI edema (in comparation with the tumor volume) } & $>=$ tumor volume & 5.0 \\
\hline & $<$ tumor volume & 12.3 \\
\hline \multirow[t]{2}{*}{ Proximity to the dura-mater } & Adjacent to the dura & 9.3 \\
\hline & Not adjacente to the dura & 14.8 \\
\hline \multirow[t]{4}{*}{ Lobar location } & Frontal & 10.0 \\
\hline & Parietal & 17.0 \\
\hline & Temporal & 8.4 \\
\hline & Occipital & 31.0 \\
\hline \multirow[t]{2}{*}{ Hemispheric location } & Left & 13.6 \\
\hline & Right & 4.3 \\
\hline \multirow[t]{2}{*}{ Tumor size (cm in axial plane) } & $>=5$ & 7.7 \\
\hline & $<5$ & 13.3 \\
\hline \multirow[t]{2}{*}{ Gliosarcoma type } & Primary & 11.1 \\
\hline & Secundary & 9.0 \\
\hline \multirow[t]{2}{*}{ Macroscopy } & Glioblastoma & 16.3 \\
\hline & Meningioma & 11.0 \\
\hline \multirow[t]{2}{*}{ Type of resection } & Parcial & 10.0 \\
\hline & Radical & 10.8 \\
\hline \multirow[t]{4}{*}{ Adjuvant treatment } & None & 5.7 \\
\hline & CT & 11.7 \\
\hline & $\mathrm{RT}+\mathrm{CT}$ & 12.7 \\
\hline & $\mathrm{RT}$ & 19.0 \\
\hline \multirow[t]{2}{*}{ Metastization } & Yes & 9.0 \\
\hline & No & 11.2 \\
\hline
\end{tabular}

Analyzing the survival according to the necrosis in MRI, patients with necrosis equal to or greater than $50 \%$ of the tumor volume obtained lower survival than those with necrosis of less than $50 \%$ of the tumor volume (9.8 and 12.1 months respectively). Patients with mean midline shift equal to or greater than $0.5 \mathrm{~cm}$ survived less than those with shift less than $0.5 \mathrm{~cm}$ ( 7.6 months and 16.3 respectively). Patients with MRI edema equal to or greater than tumor volume had a much lower survival rate than those with edema less than tumor volume (5.0 and 12.3 months respectively). Tumors adjacent to the dura were associated with lower survival rates (9.3 months versus 14.8 months for non-adherents). A parietal lobe localization acquired the best survival (17.0 months) and a shorter one for temporal location (8.4 months), as well as left hemisphere lesions obtained better survival (13.6 months) than lesions in the right hemisphere ( 4.3 months). Lesions with a size equal to or greater than $5 \mathrm{~cm}$ had lower survival rates compared to those of less than $5 \mathrm{~cm}$ (7.7 and 13.3 months respectively).

Secondary type gliosarcomas were associated with worse survivals (9.0 months) when compared to the primary ones (11.1 months). In the secondary ones, the survival from the diagnosis of glioblastoma, was of 20.8 months.

Macroscopy similar to meningioma obtained inferior survival (11.0 months) to that similar to glioblastoma (16.3 months).

Partial resection was associated with worse survival (10.0 months) than radical removal (10.8 months). Survival according to the adjuvant treatment was 5.7 months in patients not submitted to any adjuvant treatment, 11.7 
months undergoing chemotherapy alone, 12.7 months undergoing radiotherapy+chemotherapy and 19 months undergoing radiotherapy alone (Graph 2).

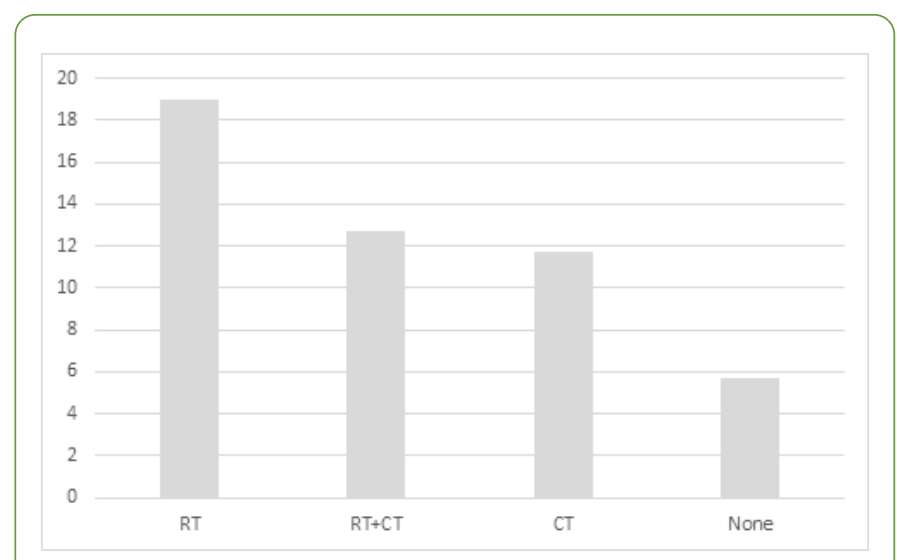

Graph 2 Survival (months) according to adjuvant treatment.

Metastasis was associated with a 9-month survival compared to 11.2 months for cases where it did not occur.

Of all the variables analyzed, those with statistical significance were the mass effect in the imaging exams $(p=0.023)$ and the hemispheric location $(p=0.015)$.

\section{Discussion}

The origin of gliosarcomas is controversial. Although the glial component originates in astrocytes, the pathogenesis of the sarcomatous component is much more doubtful [11-19]. Initially, the most accepted theory was based on the hyperplastic transformation of tumoral vessels induced by malignant glial cells [13-16]. Subsequently, with the introduction of vascular markers this theory lost importance. Currently, the most accepted theory for the pathogenesis of primary gliosarcomas is based on a monoclonal origin of its two components, either through a common percussive cell [13] or with the sarcomatous component originating through aberrant mesenchymal differentiation of glioma $[2,13,14]$. This theory is corroborated by a similar genetic profile between the two components [2].

As epidemiological features, gliosarcomas constitute $0.48 \%$ of all intracranial tumors [10] and $1.8-10 \%$ of glioblastomas $[1,6,10,13,15,17,20,21]$. In the present series, the value falls within the referred range (5\% of all glioblastomas). Like glioblastomas, they have a predilection for more advanced ages, with a mean between the 6th and 7th decades of life and the male gender with a male: female ratio of 1.4-1.8:1 [2,14]. However, this tumor is also described in the pediatric age with a peak incidence in childhood [2].

In this series, the pediatric age was not included but the gliossarcomas obtained a mean age of onset of 56 years.

An increase in the incidence of both primary and secondary gliosarcomas has been observed in Braga Hospital in the recent years, which has also been observed in some published cases. It is thought that the increased incidence of secondary gliosarcomas may be related to more effective approaches and treatment in patients with glioblastomas, increasing their survival [12].

The most frequent localization of these tumors is in the temporal lobe, as in this case series $[10,11]$. Other lobes in decreasing order of frequency are: frontal, parietal and occipital $[17,18]$. There are only 3 cases described in the literature with an infratentorial location [18].

The clinical features depend on the location of the tumor and are similar to those of glioblastomas. The symptoms most frequently described in the literature are seizures, focal neurological deficits, and others, associated with intracranial hypertension $[2,10]$.

The imaging characteristics are variable. They may present as lesions with large central necrotic areas and heterogeneous contrast uptake, similar to a glioblastoma, or as hyperdense lesions, with well-defined margins and homogeneous contrast uptake, similar to a meningioma $[2,14]$. An imaginary difference between gliosarcomas and meningiomas is that the gliosarcomas when evaluated in the bone window do not present a broad base or destructive reactions in the bone [20]. They often present a marked peritumoral edema $[2,11,14,20]$. In this series, lesions located in the left hemisphere were associated with better survival, which may be explained by the presence of more relevant symptomatology as a change of language, leading to earlier diagnosis and treatment. Lesions with a midline shift of less than $0.5 \mathrm{~cm}$ were also associated with a better survival, which may be explained by the lower degree of aggression, with a lower effect on the patient's clinical condition and consequent better tolerance to surgical treatment and complementary adjuvants.

As regards the macroscopic aspect, the two distinct types already mentioned are described. Studies have reported a correlation between imaging and macroscopic appearance, while others conclude the opposite [14].

In histological terms, hematoxylin and eosin staining shows the presence of two distinct cell populations. One formed predominantly of astrocytes with necrotic areas fulfilling the criteria of glioblastoma and another one with fusiform cells, suggestive sarcoma. With GFAP (Glial Fibrillary Acid Protein) only the glial component is stained, with very thin capture in the sarcomatous component. Cervoni and Salvati correlated the higher prevalence of the sarcomatous component found in histology with firm lesions and well-defined margins [11].

Unlike glioblastomas, the metastatic capacity of gliosarcomas is well known. As early as 1958, in Feigin's time, several authors reported cases of metallization with mixed elements. Subsequently, Smith and colleagues observed two cases of metastization in which metastasis consisted only of the sarcomatous component. These observations have raised the hypothesis that the potential metastasis of gliosarcomas is due to the sarcomatous component [14]. The higher propensity to metastasize also seems to be related to its frequent location in the temporal lobe in the proximity of the hard and venous sinuses [7]. The main metastatic sites are lung $(72 \%)$, liver (41\%) and lymph nodes (18\%) [6]. Other sites 
reported are: spleen, adrenal, kidney, oral mucosa, skin, bone marrow, skeleton and spinal cord [14]. In this case the only case metastasized to the cerebral and spinal cord leptomeninges.

The therapeutic approach, doesn't have a well-defined guideline. The first reviews published in the literature considered a set of clinical and biological similarities with glioblastomas, and since then they have been treated through the same protocols, with maximum surgical removal followed by radiotherapy and chemotherapy $[5,10,12]$. In Braga Hospital, the protocol used to treat gliosarcomas was the Stupp protocol, used in high grade gliomas. As radiotherapy and chemotherapy are well established in the treatment of glioblastomas, they also end up being used in the treatment of gliossarcomas. However, its use in gliosarcomas is not well understood [14]. Some published articles did not obtain benefit in the survival with the use of the protocol of chemotherapy and radiotherapy used in glioblastomas $[11,16]$. A 2012 study reports that therapeutic modalities with benefit in gliosarcomas survival are aggressive local treatment, including maximum surgical removal and radiosurgery [16]. Since methylation of the MGMT (O6-methylguanine-DNA methyltransferase promoter) gene is more frequent in glioblastomas than in gliosarcomas, and this methylation is associated with a better response to chemotherapy, this may justify the in efficacy of chemotherapy in gliosarcomas. A 2016 New York study reports that the combination of temozolamide with radiotherapy alone did not gain survival benefit [3]. However, there are other recent studies that report that the best survival rates are obtained with maximum surgical removal, followed by radiotherapy and temozolamide chemotherapy [9,22-24]. In this series the best survival rates were related to radical surgical removal and isolated RT as adjuvant treatment. Another genetic factor associated with treatment resistance in gliosarcomas is the TP53 mutation associated with the epithelial-mesenchymal transition process of the sarcomatous component of gliosarcomas [4] (Table 4).

Table 4 Factors associated with better survival in Braga Hospital gliosarcomas.

\begin{tabular}{|l|}
\hline Patient characteristics \\
\hline$<50$ years old \\
\hline Female gender \\
\hline Karnofsky $>80$ \\
\hline Tyme symptoms to diagnosis $<1$ month \\
\hline Imaging features \\
\hline Necrosis $<50 \%$ tumor volume \\
\hline Midline shift $<0.5$ cm \\
\hline Edema $<$ tumor volume \\
\hline Tumor not adjacent to the dura \\
\hline Size $<5$ cm \\
\hline Occipital lobe \\
\hline
\end{tabular}

\begin{tabular}{|l|}
\hline Left hemisphere \\
\hline Other characteristics \\
\hline Primary type \\
\hline Macroscopy similar to glioblastoma \\
\hline Radical resection \\
\hline Adjuvant RT \\
\hline Non metastization \\
\hline
\end{tabular}

In the Braga hospital, secondary gliosarcomas were treated similarly to a recurrent glioblastoma. The main treatment failure is local recurrence, occurring in this series after an average of 6.5 months. Regarding the time for the appearance of secondary gliosarcomas, 9.56 months after the diagnosis of glioblastoma has been described in the literature [12]. In this series, the mean time was 13.6 months.

The prognosis of gliosarcomas, such as that of glioblastomas, is poor. A study by Kozak et al. reports a worse prognosis in patients with gliosarcoma when compared to glioblastomas. Other studies report that there are no statistically significant differences between the two [2]. The mean survival of untreated gliosarcoma is 4 months [2], in patients submitted to adjuvant treatment, the survival varies between 6.25 and 11.5 months [2,14]. In this series, the median survival was 11.1 months.

Gliosarcomas with the imaging appearance of meningioma appear to be associated with a better prognosis $[2,14]$. Regarding the macroscopic appearance similar to meningioma, in some articles it is associated with a better prognosis, since as they are firm and with cleavage plane, they allow a greater rate of surgical removal. However, other studies do not reveal a difference in survival with a statistically significant value [12]. In this series, the macroscopic appearance of glioblastoma was associated with better survival.

In secondary gliosarcomas, survival is in some studies lower than primary, with an average survival time after the diagnosis of gliosarcoma of 4.4 months and after the initial diagnosis of glioblastoma of 12.6 months [12]. In this series survival was 9.0 months after the diagnosis of secondary gliosarcoma and 20.8 months since the diagnosis of glioblastoma. Other studies, such as Perry's, report better survival in patients with secondary gliosarcomas [12]. In this series, the secondary ones obtained worse survivals (8.2 compared to 10 months of primary ones). Our study has several limitations, namely the reduced number of the sample and the fact that it is retrospective, having obtained only two results with a statistically significant value.

\section{Conclusion}

The data of this series generally reflects those described in the scientific literature. Due to the rarity of these tumors and their short survival, there are insufficient data for the creation of specific therapeutic protocols. Larger cases, through multicentric studies, will be fundamental to obtain a greater 
number of results with statistically significant value. However, since gliosarcomas have specific characteristics that distinguish them from glioblastomas, these tumors may benefit from a specific treatment. This sample presents some factors with effect on the survival and that can be taken into account in the approach of these tumors. There are also two factors with influence on survival and statistically significant value: midline shift less than $0.5 \mathrm{~cm}$ and location in the left hemisphere.

\section{References}

1. Andaloussi-Saghir K, Oukabli M, El-Marjany M, Sifat H, Hadadi K, et al. (2011) Secondary gliosarcoma after the treatment of primary glioblastoma multiforme. N Am J Med Sci 3: 527-530.

2. Biswas A, Kumar N, Kumar P, Vasishta RK, Gupta K, et al. (2011) Primary gliosarcoma--clinical experience from a regional cancer centre in north India. Br J Neurosurg 25: 723-729.

3. Castelli J, Feuvret L, Haoming QC, Biau J, Jouglar E, et al. (2016) Prognostic and therapeutic factors of gliosarcoma from a multiinstitutional series. J Neurooncol 129: 85-92.

4. Cho SY, Park C, Na D, Han JY, Lee J, et al. (2017) High prevalence of TP53 mutations is associated with poor survival and an EMT signature in gliosarcoma patients. Exp Mol Med 49: e317.

5. Damodaran O, Van Heerden J, Nowak AK, Bynevelt M, McDonald $K$, et al. (2013) Clinical management and survival outcomes of gliosarcomas in the era of multimodality therapy. Journal of Clinical Neuroscience: Official Journal of the Neurosurgical Society of Australasia 21: 478-481.

6. Dawar R, Fabiano AJ, Qiu J, Khushalani NI (2013) Secondary gliosarcoma with extra-cranial metastases: A report and review of the literature. Clinl Neurol Neurosurg 115: 375-380.

7. Demirci S, Akalin T, Islekel S, Ertan Y, Anacak Y (2008) Multiple spinal metastases of cranial gliosarcoma: a case report and review of the literature. J Neurooncol 88: 199-204.

8. Ekici M, Bulut T, Tucer B, Kurtsoy A (2011) analysis of the mortality probability of preoperative MRI features in malignant astrocytomas. Turkish Neurosurgery 3: 271-279.

9. Frandsen J, Orton A, Jensen R, Colman H, Cohen AL, et al. (2017) Patterns of care and outcomes in gliosarcoma: an analysis of the National Cancer Database. JNS, pp: 1-6.

10. Guney Y, Hicsonmez A, Yilmaz S, Adas YG, Andrieu MN (2010) Gliosarcoma: A study of four cases. Rare Tumors 2: e37.

11. Han SJ, Yang I, Ahn BJ, Otero JJ, Tihan T, et al. (2010) Clinical characteristics and outcomes for a modern series of primary gliosarcoma patients. Cancer 116: 1358-1366.
12. Han SJ, Yang I, Otero JJ, Ahn BJ, Tihan T, et al. (2010) Secondary gliosarcoma after diagnosis of glioblastoma: clinical experience with 30 consecutive patients. J Neurosurg 112: 990-996.

13. Han SJ, Yang I, Tihan T, Chang SM, Parsa AT (2010) Secondary gliosarcoma: A review of clinical features and pathological diagnosis. J Neurosurgery 112: 26-32.

14. Han SJ, Yang I, Tihan T, Prados MD, Parsa AT (2010) Primary gliosarcoma: key clinical and pathologic distinctions from glioblastoma with implications as a unique oncologic entity. J Neurooncol 96: 313-320.

15. Kozak KR, Mahadevan A, Moody JS (2009) Adult gliosarcoma: Epidemiology, natural history, and factors associated with outcome. Neuro Oncol 11: 183-191.

16. Lee D, Kang SY, Suh YL, Jeong JY, Lee Jl, et al. (2012) Clinicopathologic and genomic features of gliosarcomas. J Neurooncol 107: 643-650.

17. Moiyadi A, Sridhar E, Jalali R (2010) Intraventricular gliosarcoma: Unusual location of an uncommon tumor. J Neurooncol 96: 291-294.

18. Moon SK, Kim EJ, Choi WS, Ryu CW, Park BJ, et al. (2010) Gliosarcoma of the cerebellar hemisphere: a case report and review of the literature. Korean Journal of Radiology: Official Journal of the Korean Radiological Society 11: 566-570.

19. Pakos EE, Goussia AC, Zina VP, Pitouli EJ, Tsekeris PG (2005) Multi-focal gliosarcoma: $A$ case report and review of the literature. J Neurooncol 74: 301-304.

20. Parekh HC, O'Donovan DG, Sharma RR, Keogh AJ (1995) Primary cerebral gliosarcoma: Report of 17 cases. $\mathrm{Br} J$ Neurosurg 9: 171-178.

21. Rizvi S, Asghar AH, Mehboob J (2010) Gliosarcoma: A rare variant of glioblastoma multiforme. J Pak Medical Assoc 60: 773-775.

22. Singh G, Das KK, Sharma P, Guruprasad B, Jaiswal S, et al. (2015) Cerebral gliosarcoma: Analysis of 16 patients and review of literature. Asian J Neurosurg 10: 195-202.

23. Zhang G, Huang S, Zhang J, Wu Z, Lin S, et al. (2016) Clinical outcome of gliosarcoma compared with glioblastoma multiforme: a clinical study in Chinese patients. J Neurooncol 127: 355-362.

24. Winkler PA, Buttner A, Tomezzoli A, Weis S (2000) Histologically repeatedly confirmed gliosarcoma with long survival: Review of the literature and report of a case. Acta Neurochirurgica 142: 91-95. 